\title{
NECESSARY OPTIMALITY CONDITIONS FOR NONSMOOTH VECTOR OPTIMIZATION PROBLEMS
}

\author{
DAVIDE LA TORRE \\ University of Milan, Department of Economics \\ via Conservatorio,7, 20122, Milano, Italy \\ E-mail: davide.latorre@unimi.it \\ Received September 1, 2002; revised February 13, 2003
}

\begin{abstract}
In this paper we introduce a notion of generalized derivative for nonsmooth vector functions in order to obtain necessary optimality conditions for vector optimization problems. This definition generalizes to the vector case the notion introduced by Michel and Penot and extended by Yang and Jeyakumar. This generalized derivative is contained in the Clarke subdifferential and then the corresponding optimality conditions are sharper than the Clarke's ones.
\end{abstract}

Key words: vector optimization, generalized derivatives

\section{INTRODUCTION}

A function $f: \mathbb{R}^{n} \rightarrow \mathbb{R}^{m}$ is said to be locally Lipschitzian (or of class $C^{0,1}$ ) at $x_{0} \in \mathbb{R}^{n}$ when there exist constants $K_{x_{0}}$ and $\delta_{x_{0}}$ such that

$$
\left\|f\left(x_{1}\right)-f\left(x_{2}\right)\right\| \leq K_{x_{0}}\left\|x_{1}-x_{2}\right\|
$$

$\forall x_{1}, x_{2} \in \mathbb{R}^{n},\left\|x_{1}-x_{0}\right\|<\delta_{x_{0}},\left\|x_{2}-x_{0}\right\|<\delta_{x_{0}}$. For this type of functions, Rademacher theorem states that $f$ is almost everywhere differentiable (in the sense of Lebesgue measure). Then the first order Clarke subdifferential of $f$ at $x_{0}$, denoted by $\partial f\left(x_{0}\right)$ is defined as ([2])

$$
\partial f\left(x_{0}\right)=\operatorname{cl} \operatorname{conv}\left\{l=\lim \nabla f\left(x_{k}\right), x_{k} \rightarrow x_{0}, \nabla f\left(x_{k}\right) \text { exists }\right\}
$$

where $\operatorname{cl} \operatorname{conv}\{\ldots\}$ is the closure of the convex hull of all limit points. Similarly for a function $f: \mathbb{R}^{n} \rightarrow \mathbb{R}^{m}$ of class $C^{1,1}$, that is a differentiable function with 
a locally Lipschitzian jacobian, the second order Clarke subdifferential of $f$ at $x_{0}$, denoted by $\partial^{2} f\left(x_{0}\right)$, is defined as

$$
\partial^{2} f\left(x_{0}\right)=\operatorname{cl} \operatorname{conv}\left\{l=\lim \nabla^{2} f\left(x_{k}\right), x_{k} \rightarrow x_{0}, \nabla^{2} f\left(x_{k}\right) \text { exists }\right\} .
$$

Thus $\partial^{2} f\left(x_{0}\right)$ is a subset of the finite dimensional space $L\left(\mathbb{R}^{n} ; L\left(\mathbb{R}^{n} ; \mathbb{R}^{m}\right)\right)$ of linear operators from $\mathbb{R}^{n}$ to the space $L\left(\mathbb{R}^{n} ; \mathbb{R}^{m}\right)$ of linear operators from $\mathbb{R}^{n}$ to $\mathbb{R}^{m}$. By the previous construction $\partial^{2} f\left(x_{0}\right)$ is a nonempty convex compact set of the space $L\left(\mathbb{R}^{n} ; L\left(\mathbb{R}^{n} ; \mathbb{R}^{m}\right)\right)$ and the set valued map $x \rightarrow \partial^{2} f(x)$ is upper semicontinuous. Let $u \in \mathbb{R}^{n}$; in the following we will denote by $L u$ the value of a linear operator $L: \mathbb{R}^{n} \rightarrow \mathbb{R}^{m}$ at the point $u \in \mathbb{R}^{n}$ and by $H(u, v)$ the value of a bilinear operator $H: \mathbb{R}^{n} \times \mathbb{R}^{n} \rightarrow \mathbb{R}^{m}$ at the point $(u, v) \in \mathbb{R}^{n} \times \mathbb{R}^{n}$. So we will set

$$
\partial f\left(x_{0}\right)(u)=\left\{L u: L \in \partial f\left(x_{0}\right)\right\}
$$

and

$$
\partial f\left(x_{0}\right)^{2}(u, v)=\left\{H(u, v): H \in \partial^{2} f\left(x_{0}\right)\right\} .
$$

Some important properties are listed in the following [2].

- Mean value theorem. Let $f$ be of class $C^{0,1}$ and $a, b \in \mathbb{R}^{n}$, then

$$
f(b)-f(a) \in \operatorname{cl} \operatorname{conv}\{\partial f(x)(b-a): x \in[a, b]\},
$$

where $[a, b]=\operatorname{conv}\{a, b\}$.

- Taylor expansion. Let $f$ be of class $C^{1,1}$ and $a, b \in \mathbb{R}^{n}$, then

$$
f(b)-f(a) \in f^{\prime}(a)(b-a)+\frac{1}{2} \operatorname{cl} \operatorname{conv}\left\{\partial^{2} f(x)(b-a, b-a): x \in[a, b]\right\} .
$$

In [2], Guerraggio and Luc have given necessary and sufficient optimality conditions for $C^{1,1}$ vector optimization problems, expressed by means of Clarke subdifferential. However, in literature, several alternative definitions for generalized subdifferentials has been proposed for the scalar case including, in particular, the Michel-Penot subdifferential ([8]). The Michel-Penot subdifferential of a locally Lipschitzian function at a point is a nonempty, convex, compact set, contained in the Clarke subdifferential. The smallness of the Michel-Penot subdifferential makes the corresponding results, like optimality conditions, sharper than the Clarke's ones. In [9] Yang and Jeyakumar used the notion of Michel-Penot subdifferential for $C^{1,1}$ scalar functions, proving also second order optimality conditions.

The aim of this paper is to generalize to the vector case the notions of Michel-Penot subdifferential and Yang-Jeyakumar subdifferential (sections 2 and 3 ) and to use them in order to obtain necessary optimality conditions for nonsmooth vector optimization problems involving $C^{1,1}$ data (section 4). 


\section{PRELIMINARY DEFINITIONS AND RESULTS}

Let $f: \mathbb{R}^{n} \rightarrow \mathbb{R}$ be a $C^{0,1}$ function at $x_{0} \in \mathbb{R}^{n}$. For such a function, the definition of Michel-Penot generalized derivative $\bar{f}_{M}^{\prime}$ at $x_{0}$ in the direction $u \in \mathbb{R}^{n}$ is given by ([9])

$$
\bar{f}_{M}^{\prime}\left(x_{0} ; u\right)=\sup _{z \in \mathbb{R}^{n}} \limsup _{s \downarrow 0} \frac{f\left(x_{0}+s u+s z\right)-f\left(x_{0}+s z\right)}{s} .
$$

Now let $f: \mathbb{R}^{n} \rightarrow \mathbb{R}^{m}$ be a $C^{0,1}$ vector function at $x_{0} \in \mathbb{R}^{n}$. We can define a generalized derivative at $x_{0} \in \mathbb{R}^{n}$ in the sense of Michel-Penot as follows

$$
f_{M}^{\prime}\left(x_{0} ; u\right)=\left(\bigcup_{z \in \mathbb{R}^{n}} A(z)\right)^{c},
$$

where

$$
A(z)=\left\{l=\lim _{k \rightarrow+\infty} \frac{f\left(x_{0}+s_{k} u+s_{k} z\right)-f\left(x_{0}+s_{k} z\right)}{s_{k}}, s_{k} \downarrow 0\right\} .
$$

and ()$^{c}$ denote the closure of the set. It is trivial to prove that the previous set is nonempty and compact. The following lemma states the relations between the scalar and the vector case.

Lemma 2.1. $\overline{\xi f}_{M}^{\prime}\left(x_{0} ; u\right) \in \xi f_{M}^{\prime}\left(x_{0} ; u\right), \forall \xi \in \mathbb{R}^{m}$.

Proof. We have

$\overline{\xi f}_{M}^{\prime}\left(x_{0} ; u\right)=\sup _{z \in \mathbb{R}^{n}} \limsup _{s \downarrow 0} \frac{(\xi f)\left(x_{0}+s u+s z\right)-(\xi f)(x+s z)}{s}=\lim _{k \rightarrow+\infty} g\left(z_{k}\right)$,

where $z_{k} \in \mathbb{R}^{n}$ and

$$
g\left(z_{k}\right)=\limsup _{s \downarrow 0} \frac{(\xi f)\left(x_{0}+s u+s z_{k}\right)-(\xi f)\left(x_{0}+s z_{k}\right)}{s} .
$$

By trivial calculations and eventually by extracting subsequences, we obtain

$$
\begin{aligned}
g\left(z_{k}\right) & =\lim _{j \rightarrow+\infty} \frac{(\xi f)\left(x_{0}+s_{j, k} u+s_{j, k} z_{k}\right)-(\xi f)\left(x_{0}+s_{j, k} z_{k}\right)}{s_{j, k}} \\
& =\sum_{i=1}^{m} \xi_{i} \lim _{j \rightarrow+\infty} \frac{f_{i}\left(x_{0}+s_{j, k} u+s_{j, k} z_{k}\right)-f_{i}\left(x_{0}+s_{j, k} z_{k}\right)}{s_{j, k}} \\
& =\sum_{i=1}^{m} \xi_{i} l_{i, k}=\xi l_{k}
\end{aligned}
$$


with $l_{k} \in A\left(z_{k}\right)$. Since $l_{k} \rightarrow l$ and $l \in f_{M}^{\prime}\left(x_{0} ; u\right)$, then $\overline{\xi f}_{M}^{\prime}\left(x_{0} ; u\right) \in$ $\xi f_{M}^{\prime}\left(x_{0} ; u\right)$.

Theorem 2.1. Let $f: \mathbb{R}^{n} \rightarrow \mathbb{R}^{m}$ be a differentiable function at $x_{0} \in \mathbb{R}^{n}$. Then $f_{M}^{\prime}\left(x_{0} ; u\right)=\nabla f\left(x_{0}\right) u, \forall u \in \mathbb{R}^{n}$.

Proof. Let $l \in f_{M}^{\prime}\left(x_{0} ; u\right)$; then by the definition of $f_{M}^{\prime}$ there exist sequences $z_{k} \in \mathbb{R}^{n}$ and $s_{k} \downarrow 0$ such that

$$
\begin{aligned}
l & =\lim _{k \rightarrow+\infty} \frac{f\left(x_{0}+s_{k} u+s_{k} z_{k}\right)-f\left(x_{0}+s_{k} z_{k}\right)}{s_{k}} \\
& =\lim _{k \rightarrow+\infty} \frac{f\left(x_{0}+s_{k} u+s_{k} z_{k}\right)-f\left(x_{0}\right)+f\left(x_{0}\right)-f\left(x_{0}+s_{k} z_{k}\right)}{s_{k}} \\
& =\lim _{k \rightarrow+\infty} \frac{\nabla f\left(x_{0}\right)\left(s_{k} u+s_{k} z_{k}\right)-\nabla f\left(x_{0}\right) s_{k} z_{k}+o\left(s_{k}\right)}{s_{k}} \\
& =\nabla f\left(x_{0}\right) u+\lim _{k \rightarrow+\infty} \frac{o\left(s_{k}\right)}{s_{k}}=\nabla f\left(x_{0}\right) u .
\end{aligned}
$$

We now prove a generalized mean value theorem for $f_{M}^{\prime}$. To do this, we scalarize the function and we use a mean value theorem for scalar functions proved in [6].

Lemma 2.2. Let $A \subset \mathbb{R}^{n}$ be a closed and convex subset of $\mathbb{R}^{n}$ such that $\xi A \cap \mathbb{R}_{-} \neq \emptyset, \forall \xi \in \mathbb{R}^{n}$. Then $0 \in A$.

\section{Proof. Trivial.}

Proposition 2.1. [6] Let $f: \mathbb{R}^{n} \rightarrow \mathbb{R}$ be a $C^{0,1}$ function. Then $\forall a, b \in \mathbb{R}^{n}$, $\exists \alpha \in[a, b]$ such that

$$
f(b)-f(a) \leq \bar{f}_{M}^{\prime}(\alpha ; b-a) .
$$

Theorem 2.2. Let $f: \mathbb{R}^{n} \rightarrow \mathbb{R}^{m}$ be a given $C^{0,1}$ vector function. Then the following generalized mean value theorem holds

$$
0 \in f(b)-f(a)-\operatorname{cl} \operatorname{conv}\left\{f_{M}^{\prime}(x ; b-a): x \in[a, b]\right\} .
$$

Proof. For each $\xi \in \mathbb{R}^{m}$ we have

$$
(\xi f)(b)-(\xi f)(a) \leq \overline{\xi f}_{M}^{\prime}(\alpha ; b-a)=\xi l_{\xi}, \quad l_{\xi} \in f_{M}^{\prime}(\alpha ; b-a),
$$

where $\alpha \in[a, b]$ and then

$$
\xi\left(f(b)-f(a)-l_{\xi}\right) \leq 0, \quad l_{\xi} \in f_{M}^{\prime}(\alpha ; b-a),
$$


Necessary optimality conditions for nonsmooth vector optimization 169

$$
\xi\left(f(b)-f(a)-\operatorname{clconv}\left\{f_{M}^{\prime}(x ; b-a): x \in[a, b]\right\}\right) \cap \mathbb{R}_{-} \neq \emptyset, \forall \xi \in \mathbb{R}^{m}
$$

and the previous lemma implies

$$
0 \in f(b)-f(a)-\operatorname{cl} \operatorname{conv}\left\{f_{M}^{\prime}(x ; b-a): x \in[a, b]\right\} .
$$

The obtained result states that $f_{M}^{\prime}$ is a subset of $\partial f\left(x_{0}\right)(u)$. The next example shows that inclusion may be strict.

Theorem 2.3. Let $f: \mathbb{R}^{n} \rightarrow \mathbb{R}^{m}$ be a $C^{0,1}$ vector function at $x_{0}$. Then $f_{M}^{\prime}\left(x_{0} ; u\right) \subset \partial f\left(x_{0}\right)(u)$.

Proof. Let $l \in f_{M}^{\prime}\left(x_{0} ; u\right)$. Then there exist sequences $z_{k} \in \mathbb{R}^{n}$ and $s_{k} \downarrow 0$ such that

$$
l=\lim _{k \rightarrow+\infty} \frac{f\left(x_{0}+s_{k} u+s_{k} z_{k}\right)-f\left(x_{0}+s_{k} z_{k}\right)}{s_{k}} .
$$

So, by the upper semicontinuity of $\partial f$, we have

$$
\begin{aligned}
& \frac{f\left(x_{0}+s_{k} u+s_{k} z_{k}\right)-f\left(x_{0}+s_{k} z_{k}\right)}{s_{k}} \in \operatorname{cl} \operatorname{conv}\{\partial f(x)(u) ; \\
& \left.x \in\left[x_{0}+s_{k} z_{k}, x_{0}+s_{k} u+s_{k} z_{k}\right]\right\} \subset \partial f\left(x_{0}\right)(u)+\epsilon B,
\end{aligned}
$$

where $B$ is the unit ball of $\mathbb{R}^{m}, \forall n \geq n_{0}(\epsilon)$. So $l \in \partial f\left(x_{0}\right) u+\epsilon B$. Taking the limit when $\epsilon \rightarrow 0$, we obtain $l \in \partial f\left(x_{0}\right)(u)$.

Example 2.1. Let $f: \mathbb{R} \rightarrow \mathbb{R}^{2}, f(x)=\left(x^{2} \sin \left(\frac{1}{x}\right)+x^{2}, x^{2}\right)$. $f$ is of class $C^{0,1}$ at $x_{0}=0$ and $f_{M}^{\prime}(0 ; 1)=(0,0) \in \partial f(0)(1)=[-1,1] \times\{0\}$.

\section{A GENERALIZED DERIVATIVE FOR $C^{1,1}$ VECTOR FUNC- TIONS}

Let $f: \mathbb{R}^{n} \rightarrow \mathbb{R}^{m}$ be a $C^{1,1}$ vector function at $x_{0} \in \mathbb{R}^{n}$. Then the second order generalized derivative at $x_{0} \in \mathbb{R}^{n}$ in the directions $u, v \in \mathbb{R}^{n}$ in the sense of Michel-Penot is the following

$$
f_{M}^{\prime \prime}\left(x_{0} ; u, v\right)=\left(\bigcup_{z \in \mathbb{R}^{n}} A(z)\right)^{c},
$$

where

$$
A(z)=\left\{l=\lim _{k \rightarrow+\infty} \frac{\nabla f\left(x_{0}+s_{k} v+s_{k} z\right) u-\nabla f\left(x+s_{k} z\right) u}{s_{k}}, s_{k} \downarrow 0\right\} .
$$


It is trivial to prove that $f_{M}^{\prime \prime}\left(x_{0} ; u, v\right)$ is nonempty and compact subset and that $f_{M}^{\prime \prime}\left(x_{0} ; u, v\right)=(\nabla f(\cdot) u)_{M}^{\prime}\left(x_{0} ; v\right)$. The results given above can be extended to the second order.

Proposition 3.1. Let $f: \mathbb{R}^{n} \rightarrow \mathbb{R}^{m}$ be a $C^{1,1}$ vector function at $x_{0} \in \mathbb{R}^{n}$. Then $\overline{\xi f}_{M}^{\prime \prime}\left(x_{0} ; u, v\right) \in \xi f_{M}^{\prime \prime}\left(x_{0} ; u, v\right), \forall \xi \in \mathbb{R}^{m}$.

Proof. In fact, we have

$$
\begin{aligned}
& \overline{(\xi \nabla f(\cdot) u)}_{M}^{\prime}\left(x_{0} ; v\right)=\sup _{z \in \mathbb{R}^{n}} \limsup _{s \downarrow 0} \frac{\xi \nabla f\left(x_{0}+s v+s z\right) u-\xi \nabla f\left(x_{0}+s z\right) u}{s} \\
& =\sup _{z \in \mathbb{R}^{n}} \limsup _{s \downarrow 0} \frac{\nabla(\xi f)\left(x_{0}+s v+s z\right) u-\nabla(\xi f)\left(x_{0}+s z\right) u}{s}=\overline{\xi f}_{M}^{\prime \prime}\left(x_{0} ; u, v\right)
\end{aligned}
$$

and then the thesis follows by using lemma 2.1.

Proposition 3.2. [9] Let $f: \mathbb{R}^{n} \rightarrow \mathbb{R}$ be a $C^{1,1}$ scalar function. Then, $\forall a, b \in$ $\mathbb{R}^{n}$, there exists $\alpha \in[a, b]$ such that the following generalized Taylor formula holds

$$
f(b)-f(a)-\nabla f(a)(b-a) \leq \frac{1}{2} \bar{f}_{M}^{\prime \prime}(\alpha ; b-a, b-a), \alpha \in[a, b] .
$$

Theorem 3.1. Let $f: \mathbb{R}^{n} \rightarrow \mathbb{R}^{m}$ be a $C^{1,1}$ vector function. Then the following generalized Taylor formula holds

$$
0 \in f(b)-f(a)-\nabla f(a)(b-a)-\frac{1}{2} \operatorname{cl} \operatorname{conv}\left\{f_{M}^{\prime \prime}(x ; b-a, b-a): x \in[a, b]\right\} .
$$

Proof. For each $\xi \in \mathbb{R}^{m}$, we have

$$
(\xi f)(b)-(\xi f)(a)-\nabla(\xi f)(a)(b-a) \leq \frac{1}{2} \overline{\xi f}_{M}^{\prime \prime}(\alpha ; b-a, b-a)=\frac{1}{2} \xi l_{\xi}
$$

where $l_{\xi} \in f_{M}^{\prime \prime}(\alpha ; b-a, b-a)$. So

$$
\begin{aligned}
& \xi\left(f(b)-f(a)-\nabla f(a)(b-a)-\frac{1}{2} l_{\xi}\right) \leq 0, \quad \forall \xi \in \mathbb{R}^{m} \\
& \xi\left(f(b)-f(a)-\nabla f(a)(b-a)-\frac{1}{2} \operatorname{cl} \operatorname{conv}\left\{f_{M}^{\prime \prime}(x ; b-a, b-a)\right.\right. \\
& \quad: x \in[a, b]\}) \cap \mathbb{R}_{-} \neq \emptyset, \\
& 0 \in f(b)-f(a)-\nabla f(a)(b-a)-\frac{1}{2} \operatorname{cl} \operatorname{conv}\left\{f_{M}^{\prime \prime}(x ; b-a, b-a): x \in[a, b]\right\} .
\end{aligned}
$$


Necessary optimality conditions for nonsmooth vector optimization 171

Theorem 3.2. Let $f: \mathbb{R}^{n} \rightarrow \mathbb{R}^{m}$ be a $C^{1,1}$ vector function at $x_{0} \in \mathbb{R}^{n}$. Then $f_{M}^{\prime \prime}\left(x_{0} ; u, v\right) \subset \partial^{2} f\left(x_{0}\right)(u, v)$.

Proof. We have

$$
f_{M}^{\prime \prime}\left(x_{0} ; u, v\right)=(\nabla f(\cdot) u)_{M}^{\prime}\left(x_{0} ; v\right) \subset \partial(\nabla f(\cdot) u)\left(x_{0}\right)(v)=\partial^{2} f\left(x_{0}\right)(u, v) .
$$

The following example shows that the inclusion may be strict.

Example 3.1. Let $f$ is of class $C^{1,1}$ at $x_{0}=0$

$$
f: \mathbb{R} \rightarrow \mathbb{R}^{2}, \quad f(x)=\left(x^{4} \sin \left(\frac{1}{x}\right)+x^{4}, x^{4}\right)
$$

and

$$
f_{M}^{\prime \prime}(0 ; 1,1)=(0,0) \in \partial^{2} f(0)(1,1)=[-1,1] \times\{0\} .
$$

\section{NECESSARY OPTIMALITY CONDITIONS FOR $C^{1,1}$ VEC- TOR FUNCTIONS}

Consider the following Pareto set constrained optimization problem

$$
\min _{\mathbb{R}_{+}^{m}} f(x) \quad \text { subject to } \quad x \in X,
$$

where $X$ is a subset of $\mathbb{R}^{n}$ and $f: \mathbb{R}^{n} \rightarrow \mathbb{R}^{m}$. A point $x_{0} \in \mathbb{R}^{n}$ is called a local minimum point (local weak minimum point) of (4.1) if there exists a neighbourhood $N$ of $x_{0}$ such that no $x \in N \cap X$ satisfies $f\left(x_{0}\right)-f(x) \in \mathbb{R}_{+}^{m} \backslash\{0\}$ $\left(f\left(x_{0}\right)-f(x) \in \operatorname{int} \mathbb{R}_{+}^{m}\right)$.

We remember that the following set

$$
W F\left(X, x_{0}\right)=\left\{d: \exists s_{k} \downarrow 0, x_{0}+s_{k} d \in X\right\}
$$

is called cone of weak feasible directions to $X$ at $x_{0}$.

Lemma 4.1. Let $x_{0} \in X$ be a local weak minimum point. Then $\forall d \in$ $W F\left(X, x_{0}\right), \exists s_{k} \downarrow 0$ such that $\nabla f\left(x_{0}+s_{k} d\right) d \notin-\operatorname{int} \mathbb{R}_{+}^{m}$.

Proof. Ab absurdo, $d \in W F\left(X, x_{0}\right)$ such that $\forall s_{k} \downarrow 0, \nabla f\left(x_{0}+s_{k} d\right) d \in$ $-\operatorname{int} \mathbb{R}_{+}^{m}$. Let $s_{k} \downarrow 0$ such that $x_{0}+s_{k} d \in X$. By the mean value theorem we have

$$
f_{i}\left(x_{0}+s_{k} d\right)-f_{i}\left(x_{0}\right)=\nabla f_{i}\left(x_{0}+\alpha_{i, k} d\right) d<0,
$$

if $k$ is large enough. This contradicts the local optimality of $x_{0}$. 
Theorem 4.1. Let $f$ be a $C^{1,1}$ vector function. If $x_{0}$ is local weak minimum point then

$$
f_{M}^{\prime \prime}\left(x_{0} ; d, d\right) \not \subset-\operatorname{int} \mathbb{R}_{+}^{m},
$$

$\forall d \in W F\left(X, x_{0}\right), \nabla f\left(x_{0}\right) d \in-\left(\mathbb{R}_{+}^{m} \backslash \operatorname{int} \mathbb{R}_{+}^{m}\right)$.

Proof. Ab absurdo, suppose there exists some $d \in W F\left(X, x_{0}\right)$ with $\nabla f\left(x_{0}\right) d \in$ $-\left(\mathbb{R}_{+}^{m} \backslash\right.$ int $\left.\mathbb{R}_{+}^{m}\right)$ and $f_{M}^{\prime \prime}\left(x_{0} ; d, d\right) \subset-$ int $\mathbb{R}_{+}^{m}$. Let $s_{k}$ be a sequence that satisfies the previous lemma. Taking the suitable subsequence if necessary, we have

$$
s_{k}^{-1}\left(\nabla f\left(x_{0}+s_{k} d\right) d-\nabla f\left(x_{0}\right) d\right) \rightarrow l \in f_{M}^{\prime \prime}\left(x_{0} ; d, d\right) \subset-\operatorname{int} \mathbb{R}_{+}^{m} .
$$

Thus, the assumption $\nabla f\left(x_{0}\right) d \in-\left(\mathbb{R}_{+}^{m} \backslash \operatorname{int} \mathbb{R}_{+}^{m}\right)$ implies that

$$
\nabla f\left(x_{0}+s_{k} d\right) d \in \nabla f\left(x_{0}\right) d-\operatorname{int} \mathbb{R}_{+}^{m} \subset-\left(\mathbb{R}_{+}^{m} \backslash \operatorname{int} \mathbb{R}_{+}^{m}\right)-\operatorname{int} \mathbb{R}_{+}^{m}=-\operatorname{int} \mathbb{R}_{+}^{m} .
$$

Corollary 4.1. Let $X=\mathbb{R}^{n}$ and $f$ be a $C^{1,1}$ vector function. If $x_{0}$ is a local weak minimum point, then

$$
f_{M}^{\prime \prime}\left(x_{0} ; d, d\right) \not \subset-\operatorname{int} \mathbb{R}_{+}^{m},
$$

$\forall d \in S^{1}, \nabla f\left(x_{0}\right) d \in-\left(\mathbb{R}_{+}^{m} \backslash \operatorname{int} \mathbb{R}_{+}^{m}\right)$.

Proof. It is trivial, recalling $W F\left(\mathbb{R}^{n}, x_{0}\right)=\mathbb{R}^{n}$.

So theorem 5.1 in [2] follows from corollary 4.1.

Corollary 4.2. Let $X=\mathbb{R}^{n}$ and $f$ be a $C^{1,1}$ vector function. If $x_{0}$ is a local weak minimum point then

$$
\partial^{2} f\left(x_{0}\right)(d, d) \not \subset-\operatorname{int} \mathbb{R}_{+}^{m},
$$

$\forall d \in S^{1}, \nabla f\left(x_{0}\right) d \in-\left(\mathbb{R}_{+}^{m} \backslash \operatorname{int} \mathbb{R}_{+}^{m}\right)$.

Proof. It is trivial, recalling $f_{M}^{\prime \prime}\left(x_{0} ; d, d\right) \subset \partial^{2} f\left(x_{0}\right)(d, d)$.

Example 4.1. Let $f: \mathbb{R} \rightarrow \mathbb{R}^{2}, f(x)=\left(x^{4} \sin \left(\frac{1}{x}\right)-\frac{x^{2}}{4},-x^{2}\right)$. The point $x_{0}=0$ is not a local weak minimum point. We have $f_{M}^{\prime \prime}(0 ; d, d)=\left(-\frac{d^{2}}{2},-2 d^{2}\right) \in$ -int $\mathbb{R}_{+}^{2}$ (the necessary condition is not satisfied) but

$$
\partial^{2} f(0)(d, d)=\left[-\frac{3 d^{2}}{2}, \frac{d^{2}}{2}\right] \times\left\{-2 d^{2}\right\}
$$

i.e., the necessary condition is satisfied. 


\section{CONCLUSIONS}

The study of the class of $C^{1,1}$ functions has been renewed since the work of Hiriart-Urruty, Strodiot and Hien Nguyen [5]. The need for investigate these functions, as pointed out in several papers on this topic (see [2] and the references therein) comes from the fact that several problems of applied mathematics including variational inequalities, semi-infinite programming, iterated local minimization, etc. involve differentiable functions with no hope to be twice differentiable. The notion of generalized derivative for this class of function is crucial in order to derive second order optimality conditions. For the scalar case several definitions have been proposed including, in particular, the Michel-Penot derivative and the Yang-Jeyakumar derivative. In this paper these notions have been extended to the vector case and then used for proving necessary optimality conditions for vector problems. These generalized derivatives are subsets of Clarke subdifferentials and this is important for establishing the "best" optimality conditions for nonsmooth vector problems. Example 4.1 shows that these new conditions can be used when Clarke's ones fail.

\section{REFERENCES}

[1] Ben-Tal A. and Zowe J. Directional derivatives in nonsmooth optimization. Journal of Optimization Theory and Applications, 47(4), 483 - 490, 1985.

[2] Guerraggio A. and Luc D.T. Optimality conditions for $C^{1,1}$ vector optimization problems. Journal of Optimization Theory and Applications, 109(3), 615 - 629, 2001.

[3] La Torre D. and Rocca M. $C^{k, 1}$ functions and Riemann derivatives. Real Analysis Exchange, 25, 743 - 752, 1999-2000.

[4] Luc D.T. Taylor's formula for $C^{k, 1}$ functions. SIAM Journal on Optimization, 5, 659 $669,1995$.

[5] Hiriart-Urruty J.B., Strodiot J.J. and Hien Nguyen V. Generalized Hessian matrix and second order optimality conditions for problems with $C^{1,1}$ data. Applied Mathematics and Optimization, 11, 43 - 56, 1984.

[6] Borwein J.M., Fitzpatrick S.P. and Giles J.R. The differentiability of real functions on normed linear space using generalized subgradients. Journal of Mathematical Analysis and Applications, 128, 512 - 534, 1987.

[7] Liu L. and Krisek M. Second order optimality conditions for nondominated solutions of multiobjective programming with $c^{1,1}$ data. Applications of Mathematics, 45(5), 381 $-397,2000$.

[8] Michel P. and Penot J.P. Calcul sous-différential pour des fonctions lischitziennes an nonlipschitziennes. Comptes Rendus de l'Academie des Sciences Paris, 298, 269 - 272, 1984.

[9] Yang X.Q. and Jeyakumar V. Generalized second-order directional derivatives and optimization with $C^{1,1}$ functions. Optimization, 26, 165 - 185, 1992. 
Būtinos neglodžių vektorių optimizavimo uždavinių optimalios sąlygos

D. La Torre

Straipsnyje ivvedama apibendrintos išvestinès sąvoka neglodžioms vektor-funkcijoms, kad galima būtų gauti optimalumo sąlygas vektorių optimizavimo uždaviniams. Šis apibrěžimas apibendrina Michel ir Penot įvestas sąvokas, kurias išplètė Yang ir Jeyakumar. Išvestinès apibendrinimas įeina į Clarke subdiferencialą, tačiau optimalumo sąlygos yra jautresnès nei Clarko. 